\title{
Application of CFD Technology in the Development and Research of Internal Combustion Engine
}

\author{
Qingguo Luo ${ }^{1, a}$,Xiaoyu $\mathrm{Si}^{2, b}$ Hongtao Yin ${ }^{3, \mathrm{c}}$ \\ ${ }^{1}$ Academy of Armored Forces Engineering, Beijing 100072, China \\ ${ }^{2}$ Academy of Armored Forces Engineering, Beijing 100072, China \\ ${ }^{3}$ Academy of Armored Forces Engineering, Beijing 100072, China \\ ${ }^{a}$ lqg_zgy@163.com, bsxy63062529@126.com, 'yinht2006@126.com
}

\begin{abstract}
Keywords: CFD technology; Internal combustion engine; Application.
Abstract. In this paper, we analyzed the shortcomings of traditional technology in the development and research of internal combustion engine, introduced the principle and advantages of CFD technology. Also we elaborated the development and application of CFD technology in the development and research of internal combustion engine. At last, we made an overview of its prospect.
\end{abstract}

\section{The shortcomings of traditional technology in the development and research of internal combustion engine}

Traditional internal combustion engine design is mainly based on the experience of the concept of macro extrapolation, debugging repeatedly on the bench, contrast, and various "lumped parameter" semi-empirical analysis, methods of trial and error. Due to the limitation of conditions and techniques, computational fluid analysis is often required to abstract and simplify the calculation object. For complex nonlinear physical quantities, it is difficult to obtain satisfactory results. Although the traditional experimental method is intuitive, but it is often affected by the model size, boundary environment difference, the personal safety and accuracy of measurement, and other factors, sometimes it is not only difficult to get good results, but also because the limitation of fund input, resources enormous waste and the cycle is too long, it is unable to fully optimize the product design, also it has larger optional for performance prediction, and often presents the cost of large, long cycle, the applicability is small, the effect is not obvious, and so on.

\section{The principle and advantages of CFD technology}

The CFD method overcomes the weakness of traditional theoretical calculation and experimental measurement, the application of CFD technology for optimization design and simulation of internal combustion engine, not only provide large amount of information, low cost, short cycle, and the suitability is strong. It can be widely used in a short period of time to study a wide range of parameters, and provides guidance for the development of new type of internal combustion engines and the performance of the old internal combustion engines.

CFD technology principle. Fluid is the research object of CFD, fluid properties and flow state determine the CFD calculation model and the choice of calculation method, and the final distribution of flow field in various physical quantities. CFD problem solving process is shown in figure 1 [1]. The governing equation is a mathematical description of the physical conservation laws governing the flow of the fluid. According to the research contents, the differential equations of the continuous change of the flowing object is established, including mass conservation, momentum conservation equation, energy conservation, components and turbulent transport equation. Choose the appropriate boundary conditions is very important to accurately reflect the flow object properties, the solution of the flow field can be obtained only if the reasonable boundary conditions are given, therefore, boundary condition is a necessary condition for the solution of the CFD problem. 


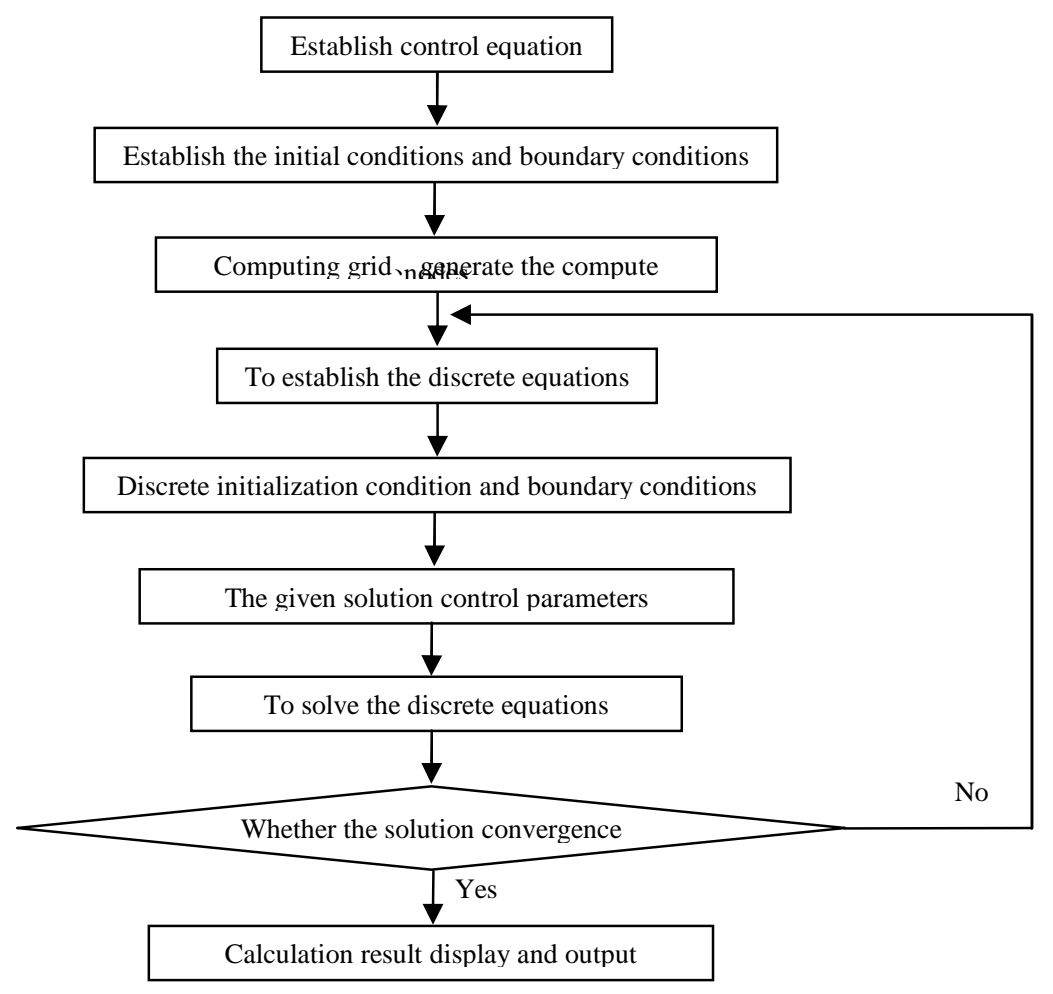

Fig.1 CFD solving process

Theoretically, the control equation in the solution domain is exact solutions, but because of the complexity of the processing problems, generally it is difficult to obtain the exact solution. Therefore, the control equation is usually dispersed in the solution domain; the partial differential equations are discredited into algebraic equations to solve the problem. To discrete control equations in the spatial domain, you must use the grid. The finer the mesh is, the closer the calculation results to the actual solution. However, restricted by computing speed and computer capacity, the grid cannot be too thin. Due to the choice of mathematical model, discrete method, calculation method and the initial boundary value conditions are different, the same physical model of the calculation results may be different, and therefore, we must select proper model and solving method according to the features of the object of study and research content.

CFD technology advantages. Compared with the traditional experiment simulation, CFD technology has a great advantage: small expenditure, less experimental model, and we can get the simulation results in a relatively short period of time, and high precision. This has laid the foundation for shortening the research period of product development. The advantages of CFD technology is summarized as follows:

(1) To save time, energy, money;

(2) Facilitate optimization design, more freedom than experimental design and more flexible;

(3) Can be used for high temperature and dangerous environment, traditional experiment is hard to get the detailed information;

(4) Conditions of repeatability, easy control, simulation process can be repeated.

\section{CFD technology in the development in the study of internal combustion engine development}

The earliest study of CFD numerical simulation in internal combustion engine can be date back to the late 1860s, R.S.Benson's work. He used the one-dimensional calculation of the gas flow in the airway to achieve success. With development of further research of internal combustion engine, the 
development of computer technology and related disciplines, CFD simulation in internal combustion engine has also experienced a development process from simple to complex.

Zero-dimensional model. At the beginning of the study, due to the development of the related discipline degree and the limits of computer development level, the work process of conventional made a simplified calculation, mainly adopted zero-dimensional model. It in time, as the only variable parameter exactly the same at various points within the system boundary, using ordinary differential equation of the state of the system was described, could predict some parameters affect the performance of internal combustion engine. However, zero-dimensional model ignores the combustion process in the nature of complex physical and chemical reaction process, and it is unable to grasp its regularity from the essence, thus cannot predict emissions generated.

Quasi-dimensional model. Quasi-dimensional model is established on the basis of the zero-dimension model, as well as zero-dimension model, it is also as the only variable in time. But in order to predict the internal combustion engine emission characteristics, developing some partition model. The combustion chamber is divided into several districts in these models, considering the nature of different physical processes in different area, and the physical parameters of each region is uniform, has nothing to do with the space coordinates. Quasi-dimensional model, to a certain extent, can reflect the relevant parameters, along with the change of the combustion chamber space for a particular type, can accurately predict the combustion process of main performance parameters, and also can predict emission performance, relative to the multi-dimensional model, and the computational cost is very low. But as with zero-dimension model, it is far from revealing the mechanism of combustion phenomenon in nature.

Multi-dimensional simulation. The working process of the internal combustion engine is extremely complex, the air intake and the compression processes have very complex dynamic behavior, which is the superposition of many complex fields, the serious influence oil and gas mixing process and combustion process. Multi-dimensional simulation for the independent variable in time and space scales, by calculating flow system space inside, can provide the distribution of the speed, pressure, temperature, concentration of components, the turbulent kinetic energy and so on, at any position at any time, , then burning, exothermic regularity, flame propagation, the pollutants generated analysis, etc., improving the existing structure of internal combustion engine provide predictive guidance, developing new type combustion system.

CFD software. The improvement of the turbulence model, the development of mathematical methods, and the introduction of high speed large capacity computer provide a good condition for the research of CFD auxiliary combustion engine. Due to the three- dimensional simulation descriptions of physical processes and boundary conditions are indeed decided close to reality, and thus have greater development potential. At present, the representative of internal combustion engine multi-dimensional numerical simulation program has the imperial college London Phonics, Star-CD, the CFX of AEA Technology company, FLUENT software companies in the United States launched the FLUENT software, KIVA developed by the United States LosAlamos National Laboratory and Austrian AVL Fire software, etc. In recent years, these software have been developed rapidly, and have been widely used all over the world, made with the theoretical results fit well with the experimental results of the calculation results, shows strong prediction ability, the internal combustion engine research, development, design and experimental research have a lot of inspiration and reference value.

\section{The application of CFD technology in internal combustion engine development research}

A lot of research on design and working process of the internal combustion engine has been carried out by scientists at home and abroad with the application of CFD technology, and a more fruitful results have been achieved.

The internal combustion engine design. Because CFD can accurately give the details of the fluid flow, thus it can be found from the quantitative analysis of the flow field of product design problems, 
according to this to optimize the design, to achieve the goal of change the traditional product design process. Ugur Kesgin [2] uses AVL Boost and application software Fire simulation studies on the internal combustion engine exhaust system, and carries on the optimization design.Wenchao Suo etc. [3] use CFD for internal combustion engine cylinder head and exhaust design, cooling and lubricating system, optimize the distribution agencies, to simulate the working process in cylinder and the power cabin air flow and heat transfer, provide strong guidance for internal combustion engine design. Maoqiang Zhu and Feiyun $\mathrm{Xu}[4]$ analyze the causes of the exhaust interference; have improved the design scheme, the new exhaust system makes the machine performance improved significantly. Gang Xu etc. [5] use the CFD analysis software such as SC/Tetra to simulate the three-dimensional flow of the cooling system, its structure has been optimized design and calculation, the coolant flow field distribution is more reasonable, improve the cooling effect. Xiaoping Bi etc. [6] use CFD to optimize the shape and size of the pipe belt type water radiator core, and reduce the flow resistance of the air side.

The exhaust process analysis. Internal combustion engine exhaust system in the gas flow is very complex, instantaneous degeneration, uneven distribution of flow system and its movement had a great influence on the properties of internal combustion engine. The gas flow CFD numerical simulation method can obtain the flow passage of pressure, velocity and turbulent kinetic energy distribution.

Air filter as the importance of the internal combustion engine intake components, its performance will affect the internal combustion engine performance, fuel economy and service life. Jia Li etc. [7] use CFD simulation computation of air filter applications such as the internal flow field, study the material, size and number of pleated filter paper and so on various factors affect the performance of air filter laws as well as the coupling mechanism between different affecting factors. Inlet is decided for the reasonable design of mixture formation, filling quantity change and combustion process of key link, and the spiral inlet consists of irregular surface, the shape is very complex, is a key problem for the inlet design. J.C.Dent and A.Chen have carried on the experiment and calculation of the bending steady flow in the intake port and used the Star-CD program to predict the affected by the shape of variable valve lift and airway flow field structure, and validated the feasibility of the simulation results. Liwei Sun etc. [8] carry on the steady state analysis of CFD to the high power diesel engine high pressure differential spiral inlet, using the results of the analysis of one-dimensional unsteady model of valve flow coefficient correction, according to the correction of the simulation model to improve the design of the inlet has a good inlet performance. Fang Gu etc. [9] use CFD technology tightly coupled catalytic converter in the exhaust system, secondary catalytic converter, main muffler and muffler parts such as numerical calculation, analysis of the flow characteristics, find out the reason of exhaust back pressure and various components, the structure is improved to get a good effect. Xiao-ping Bi etc. [10] build the establishment of a tank and exhaust flow and heat transfer calculation hydrodynamics model, using Fluent software to complete the three dimensional numerical analysis, and obtain the tanks exhaust flow field and temperature field distribution characteristics in detail and the external environment impact on exhaust temperature and wind speed, provide the basis to the research of the infrared radiation characteristics of the tank's exhaust.

Analysis of in cylinder. The requirement of increasing the internal combustion engine fuel economy and emissions promotes some new technology appeared, such as high voltage electronic control injection, injection, gasoline direct injection and exhaust gas recirculation, and so on. These technologies need people on the work process of the internal combustion engine and internal combustion engine combustion process in cylinder has a deeper understanding. CFD technology for researchers to better understand the movement process of fluid flow in cylinder, the changes of the turbulence parameters, the spatial distribution of fuel particles and progress in the combustion process, heat transfer process and the distribution of temperature field in cylinder possible.

Augusto C.M.Moraesetc is proposed based on the finite element method to solve the strategy of new numerical simulation of unsteady flow in cylinder [11]. Yuanmo Xu form Tsinghua University, etc. [12] study the internal combustion engine cylinder is commonly used in numerical simulation of 
physical model, calculation method and the grid generation technique, and analyzes the application characteristics of each model. Chuntao Shi etc. [13] study the spray, such as broken model hole nozzle flow model and based on the theory of surface WAVE instability WAVE model, KH - RT model, Huh Gosman model and LISA model as well as their application in the CFD software. Zhixia He etc. [14] study the characteristics of the eccentric needle valve when the internal hole orifice flows, the results show that low pressure backflow zone at the entrance of the nozzle on the flow of the nozzle exit had a greater influence on the distribution.

Thermal management analysis. Internal combustion engine the gas flow state in the cabin and thermal environment is very complex, if parts of the internal combustion engine hold arrangement don't listen to obstruct the flow of cooling air, resulting in cabin temperature on the high side. Kun Zhang etc. [15] use Fluent software and carry out three-dimensional simulation on the gas flow of the internal combustion engine compartment, find that the cooling gas flow is the basic reasons for internal combustion engine overheat, and put forward the improvement scheme of adding choke plate, effectively improve the models the flow of the internal combustion engine cooling gas chamber, and have solved the problem of the cabin temperature which is too high. Xiancheng Wang etc. [16] establish electric drive vehicles, such as the power cabin air flow and heat transfer calculation of physical model and mathematical model of the power cabin under different fan flow air flow field in the three-dimensional numerical simulation and analysis, and have analyzed the exhaust window where the influence of cooling effect to hold components.

The cooling system analysis. The internal combustion engine cooling system is an important auxiliary system to guarantee the normal and stable operation of internal combustion engine, heat can be taken away by the flow of cooling medium. Optimize the coolant flow field analysis is an important means to reduce cooler and fan diameter volume, reduce the fan noise and energy consumption, reduce manufacturing cost and the operation cost of vehicle. Xiaohu Tian etc. [17] use Fluent software to do some simulation studies on the air side flow field ,pressure field and temperature field of the fin type heat exchanger of the auto shutter, analysis the different structure parameters affect the performance of the fin in heat and flow. Xiaohui Huang etc. [18] establish the armored vehicles, such as the cooling air duct air turbulent flow and heat transfer of CFD calculation model, which is applied to the vehicle in the design of the cooling air duct, good results have been achieved. Xiumin Yu etc. [19] use numerical simulation technology to study the flow and heat transfer in internal combustion engine cooling system problems, including water set of single phase flow and gas liquid two phase flow, the fluid-structure coupling heat transfer, one-dimensional and three-dimensional joint simulation, hot spot content in the field. Dongsheng Xia etc. [20] use the application of grid technology and complete capitation flow model, two phase mixture to a certain type of main side of the cooling water of diesel engine cylinder liner capitation flow unsteady numerical simulation.

\section{Development and prospect}

CFD is not purely theoretical analysis, but is more approximate to experimental research, and greatly depend on some relatively simple, linear, strict mathematical analysis about problems with the original, and rely on to try and change method and test the empirical formula, it comes from practice, is one of the modern design must be a powerful tool for internal combustion engine. Because of the development of computer hardware and numerical technology improvements, CFD has become an extremely effective means of science, has huge development potential, will play a more and more important role in research of internal combustion engine. But we should also see that, at present the improvement of computing speed is more dependent on the improvement of computer speed, and CFD calculation method to improve the computation time of the effect is not obvious, needs to further improve on the calculation method of CFD. 


\section{References}

[1] Xicong Zhou, Xiaojing Liu. Small enterprises of science and technology,2007(6):191-192.In Chinese.

[2] UgurKesgin. Energy Conversion and Management, 2005 (46):2258-2287.

[3] WENchao Suo, Shu Han, Jing Zhang. Vehicle \& Power Technology,2006(1):37-4. In Chinese.

[4] Maoqiang Zhu, Yunfei Xu. The internal combustion engine,2011(4):1-5. In Chinese.

[5] Gang Xu, Shuli Jiang, Fei Dong, Shu Bai. Small internal combustion engine and motorcycle,2011(6):62-4. In Chinese.

[6] Xiaoping Bi, Hejia Li, Wenchao Suo. The internal combustion engine engineering,2010(1):97-3. In Chinese.

[7] Jia Li, Zhentao Liu, Zhongmin Liu. Journal of zhejiang university (Engineering science).2012,46(2):327-332. In Chinese.

[8] Liwei Sun, Tiexiong Su, Junfeng Xu. Journal of China Ordnance,2011,32(11):1310-1314. In Chinese.

[9] Fang Gu, Botan Liu, Hongliang Li. Journal of the internal combustion engine, 2007,25(4):359-363. In Chinese.

[10] Xiaoping Bi, Yu Yang. Journal of China Ordnance,2008,29(9):1025-1028. In Chinese.

[11] Maji Luo, Zhen Huang, Yankun Jiang. Automotive engine,2003(10):59. In Chinese.

[12] Yuanmo $\mathrm{Xu}$, Shi-jin Shuai, Yanjun Wang. Small internal combustion engine and motorcycle,2002,31(5):36-41. In Chinese.

[13] Chuntao Shi, Lixing Sun, Jianjun Liu. Journal of agricultural machinery,2007,38(2):189-194. In Chinese.

[14]Zhixia He, Detao Li, Qian Wang. Journal of mechanical engineering,2005,41(3):92-97. In Chinese.

[15] Kun Zhang, Yuzhang Wang, Xiaoyu Yang. Automotive engineering,2001,33(4):314-317. In Chinese.

[16] Xiancheng Wang, WENchao Suo, Gengyun Zhang. Journal of China Ordnance,2007,28(6):744-748. In Chinese.

[17]Xiaohu Tian, Longjian Li. Journal of tianjin polytechnic university,2007,23(2):63-66. In Chinese.

[18] Xiaohui Huang, Xiaoping Bi. Journal of China Ordnance,2008,29(11):1218-1285. In Chinese.

[19]Xiumin Yu, Haibo Chen, Hai-zhen Huang. Journal of mechanical engineering,2008,44(10):162-167. In Chinese.

[20] Dongsheng Xia, Huichen Zhang, Yan Yu. Journal of mechanical engineering,2011,47(22):167-173. In Chinese. 